\title{
Anticonvulsants for Psychiatric Disorders in Children and Adolescents: A Systematic Review of Their Efficacy
}

\author{
Chiara Davico, Carlotta Canavese, Roberta Vittorini, Marina Gandione and \\ Benedetto Vitiello*
}

Division of Child and Adolescent Neuropsychiatry, Department of Public Health and Pediatric Sciences, University of Turin, Turin, Italy

\section{OPEN ACCESS}

Edited by:

Fabio Blandini,

Fondazione Istituto Neurologico Nazionale Casimiro Mondino (IRCCS),

Italy

Reviewed by: Umberto Balottin, University of Pavia, Italy Emilio Perucca,

University of Pavia, Italy

*Correspondence: Benedetto Vitiello bvitiel/@unito.it

Specialty section:

This article was submitted to Child and Adolescent Psychiatry,

a section of the journal

Frontiers in Psychiatry

Received: 19 March 2018 Accepted: 05 June 2018

Published: 22 June 2018

Citation:

Davico C, Canavese C, Vittorini $R$, Gandione M and Vitiello B (2018)

Anticonvulsants for Psychiatric

Disorders in Children and

Adolescents: A Systematic Review of

Their Efficacy. Front. Psychiatry 9:270.

doi: 10.3389/fpsyt.2018.00270
Aim: Anticonvulsant medications are frequently used in clinical practice to treat psychiatric disorders in children and adolescents, but the evidence for their efficacy is uncertain. We conducted a systematic review of published randomized controlled trials $(R C T)$ that assessed the psychiatric benefit of anticonvulsants in patients under 18 years of age.

Method: The Medline, Scopus, Web of Science, and ClinicalTrials.gov databases were systematically searched for peer-reviewed primary publications of RCTs with a minimum of 10 patients per treatment arm through December 2017.

Results: Out of 355 identified non-duplicative publications, 24 met the inclusion criteria. Most RCTs were to treat bipolar disorder $(n=12)$ or manage recurrent aggression $(n=9)$. Few $(n=3)$ had both a multisite design and adequate statistical power. Valproate was the most frequently studied anticonvulsant $(n=15)$. Out of three placebo-controlled RCTs of valproate in bipolar disorder, none showed efficacy. In four RCTs, valproate was inferior to the antipsychotic risperidone. In several small, single-site RCTs, valproate and sulthiame were better than placebo for the management of recurrent aggression.

Conclusions: Currently available RCTs do not support the efficacy of anticonvulsants as mood stabilizers in children. There is some preliminary evidence from small RCTs of the efficacy of some anticonvulsants in the control of aggression and behavioral dyscontrol in conduct disorder, autism, and intellectual disability.

Keywords: anticonvulsants, children, psychiatric, bipolar, aggression, clinical trial

\section{INTRODUCTION}

Anticonvulsant medications have been used for decades in the treatment of psychiatric disorders. It is postulated that the biochemical mechanisms underlying their anti-seizure activity can lead also to stabilization of mood and behavior (1). In adults, valproate, carbamazepine, and lamotrigine have demonstrated efficacy as mood stabilizers in acute mania and/or as maintenance treatment of bipolar disorder to prevent recurrence (2-5). Oxcarbazepine and topiramate are also used, but without clear-cut evidence of efficacy (6-8). In addition, some anticonvulsants have anti-aggressive properties, and carbamazepine, oxcarbazepine, and phenytoin have been found to be effective in the management of recurrent impulsive aggression (9). 
In children (here intended as individuals under 18 years of age), anticonvulsants are frequently used to stabilize mood and behavior, usually in the context of bipolar disorder or other disorders that are accompanied by recurrent aggression, self-injury, or severe temper dysregulation, such as intellectual disability, autism spectrum disorder, conduct disorder, and attention deficit-hyperactivity disorder (ADHD) (10). In fact, anticonvulsants have been among the most commonly used pharmacological agents in pediatric bipolar disorder (11).

No anticonvulsant currently carries regulatory approval for pediatric use for the treatment of bipolar disorder or other psychiatric indications. Thus, anticonvulsants are used "off label" in children. Uncontrolled investigations have been indeed suggestive of efficacy (12). Uncontrolled studies, however, cannot constitute evidence of efficacy, especially in psychiatric conditions, such as mood disorders, that are characterized by high rates of spontaneous improvement and placebo effect. Only randomized controlled trials (RCTs) can demonstrate efficacy.

In order to evaluate the evidence for the efficacy of anticonvulsants in the treatment of psychiatric disorders in children, we conducted a systematic review of RCTs. The main aim was to identify which anticonvulsants, if any, have proven efficacy in the treatment of psychiatric disorders in children. According to evidence-based medicine standards, efficacy would be proven if supported by at least two independent RCTs.

\section{METHODS}

The standard methodology of systematic reviews was applied (13).

\section{Selection Criteria}

We searched for English language, peer-reviewed publications that were the primary reports of RCTs testing the efficacy of anticonvulsants in the treatment of psychiatric disorders in children. Included were all anticonvulsants with proven anticonvulsant effects and currently approved for the treatment of epilepsy. The psychiatric conditions included: mood disorders (depression and bipolar disorder), conduct disorder, recurrent aggression, $\mathrm{ADHD}$, anxiety, autism spectrum disorder, eating disorders, and tic disorders. Excluded was the use of anticonvulsants for migraine, headache, neuropathy, or pain management. Excluded were also RCTs in which the anticonvulsant was not the independent variable being tested for efficacy. A minimum sample size of 10 children randomized to each treatment group was required for inclusion. RCTs that enrolled adults, in addition to children, were included only if the study sample had a preponderance of subjects under 18 years of age.

\section{Search Mechanism}

The Medline, Web of Science, and Scopus databases were systematically searched for English language publications through December 2017. The search inputs were: "anticonvulsant and children (age 0-17 years) and psychiatric disorder or bipolar disorder or mania or depression or anxiety or aggression or autism or conduct disorder or ADHD or Tourette or eating disorder," repeated for specific anticonvulsant medication (i.e., valproate, carbamazepine, oxcarbazepine, lamotrigine, phenytoin, topiramate, gabapentin, pregabalin, levetiracetam, clonazepam, clobazam, perampanel). All searches used clinical trial as a filter. In addition, the ClinicalTrial.gov database was similarly searched for clinical trials of anticonvulsants in children for bipolar disorder, anxiety, ADHD, and autism.

\section{Review and Selection Process}

After removal of duplicates, the publication titles and abstracts were visually inspected and reviewed independently based on the selection criteria by two experts (CD and BV). Disagreements were discussed and resolved by consensus in order to arrive at an agreed upon list of RCT publications.

\section{Analysis}

A qualitative analysis of the selected studies was independently conducted, based on the study reported characteristics and outcomes. The characteristics, quality, and limitations of each selected RCT were assessed based on the published report. Disagreements between raters were resolved by discussion and consensus. When additional information was needed, the corresponding author of the report was contacted in an attempt to acquire additional information. In assessing quality of each study, the presence of the following seven elements was examined: (1) double-blind design; (2) description of randomization and masking methods to minimize assessment biases; (3) multiple clinical sites (more than one); (4) sample size of at least 40 children randomized to each treatment group to provide statistical power to detect medium treatment effect sizes; (5) sufficient exposure to treatment with respect to dosage (i.e., dose in the known therapeutic range for anticonvulsant action, supported by plasma levels when available) and duration of treatment (at least 3 weeks for acute efficacy and at least 12 weeks for efficacy of maintenance treatment); (6) acceptable sample retention ( $<25 \%$ of the randomized sample lost to follow-up); and (7) intent-to-treat analyses.

\section{RESULTS}

The initial search yielded a total of 351 non-duplicate publications; an additional 4 publications were identified through manual reference review or other sources. Of these 355 publications, 331 were excluded as not meeting the prespecified selection criteria, being 9 not in English, 11 not on anticonvulsants, 136 not for psychiatric use, 162 not RCT, 2 with too small of a sample size, and 11 secondary analyses of RCT (see Figure 1).

A total of 24 publications, each constituting the primary report of a RCT of anticonvulsant efficacy in psychiatric disorder in children were identified (see Tables 1, 2). Half of these RCTs were in the treatment of bipolar disorder, including acute control of manic/mixed episodes and chronic maintenance to prevent recurrence (see Table 3). The other most common psychiatric use was for the control of recurrent impulsive aggression, mainly in the context of a neurodevelopmental disorder, such as autism and/or intellectual disability $(n=9)$. 


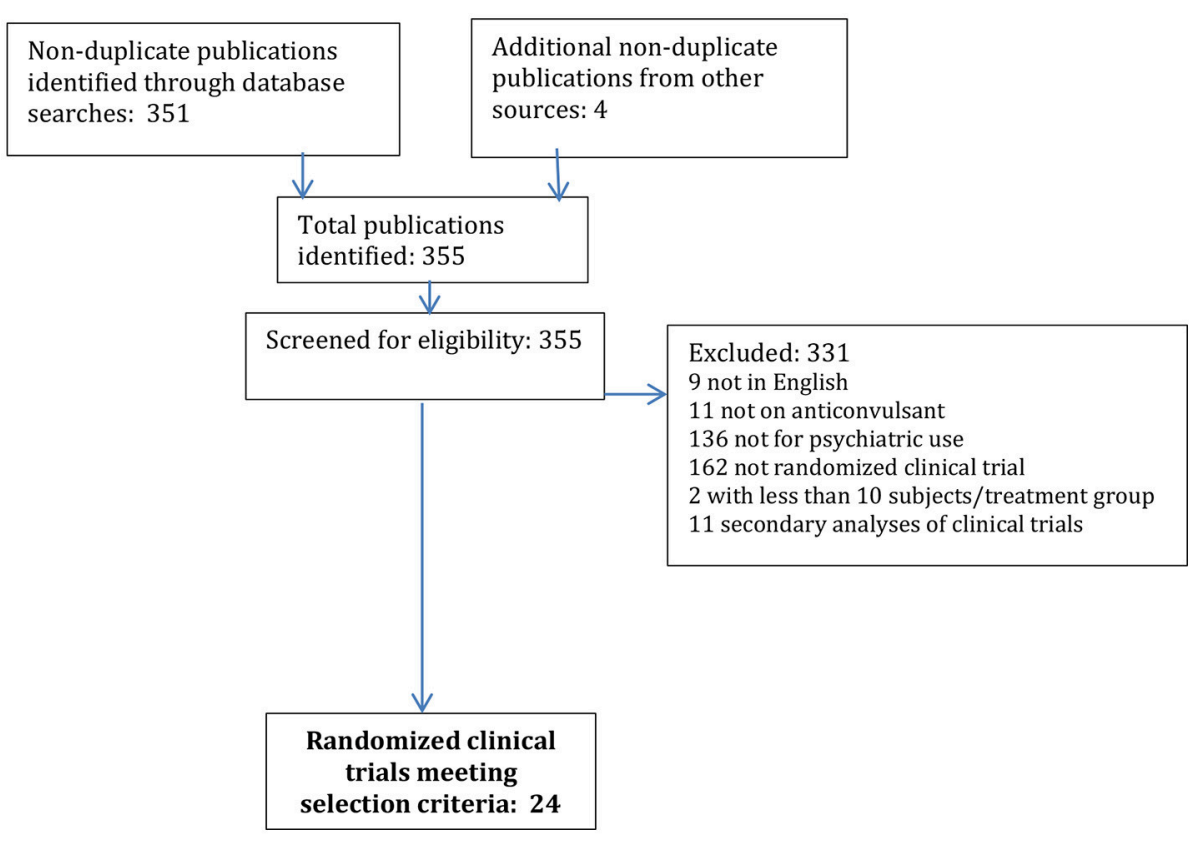

FIGURE 1 | Review flowchart.

The RCTs were conducted in double-blind conditions, except for four, which, however, employed masking methods (i.e., blinded raters) to limit ascertainment biases. Most were placebocontrolled, while four were comparative effectiveness RCTs of different active medications without a placebo control. The age of the RCT samples was mainly between 5 and 17 years. Only two RCTs included preschoolers as young as 3 years of age $(21,32)$.

Treatment exposure, with respect to adequate dosage and sufficient duration, as well as retention and statistical analyses, were considered to be satisfactory, but only five RCT involved more than one site and only five had a sample size of at least 40 subjects per treatment group (see Supplementary Table 1). Of the 24 RCTs, 13 (54\%) did not find a statistically significant difference (Tables 1, 2).

Only two RCTs were deemed to have met all the specified seven quality elements $(17,24)$, and, in particular, to have adequate sample size. Neither of these studies found the anticonvulsant medication to be better than placebo.

Most of the RCTs evaluated valproate $(n=15)$, while three tested carbamazepine (one of these studies included also valproate). The remaining anticonvulsants (oxcarbazepine, lamotrigine, levetiracetam, clonazepam, topiramate, and sulthiame) had only one or two RCTs each. Valproate was tested as a mood stabilizer in bipolar disorder in 10 RCTs and in the prevention of recurrent aggression in five RCTs. In bipolar disorder, none of the three placebo-controlled RCTs showed efficacy $(16,17,21)$. Four RCTs showed superiority of the antipsychotic risperidone over valproate (18-21). No difference was detected between valproate and lithium (14), carbamazepine (23), or quetiapine (15). Finally, one RCT conducted to test the antimanic effects of topiramate in hospitalized youths, using valproate as a comparison group, found valproate to be superior to topiramate (22).

As anti-aggressive agent, valproate showed no difference from placebo in one RCT (29) and was better than placebo in three small RCTs, one in children with autism spectrum disorder (30) and two in children with conduct disorder or $\operatorname{ADHD}(26,28)$. One RCT that compared high with low dose of valproate found superiority of the higher dose (27).

Four RCTs tested carbamazepine or oxcarbazepine in the treatment of mania, aggression, or ADHD. Two of these RCTs found no difference from placebo $(24,31)$. Another RCT found carbamazepine inferior to clonidine in ADHD (32), and in the third one there was no difference vs. valproate (23).

No evidence of efficacy emerged for lamotrigine, levetiracetam, and clonazepam. In two RCTs that were conducted more than 40 years ago in institutionalized, severely impaired subjects with intellectual disability, including both youths and adults, sulthiame, was better than placebo for controlling aggression and hyperactivity $(36,37)$.

\section{Conclusions}

This systematic review identified mostly small controlled studies with important methodological limitations and heterogeneity with respect to type of medication and clinical target. No evidence emerged for the efficacy of anticonvulsants in children with bipolar disorder. There is limited evidence for the efficacy of valproate and sulthiame for the management of aggressive behavior. For sulthiame studies were conducted in samples that included adult patients and the specific efficacy in children cannot be estimated. 


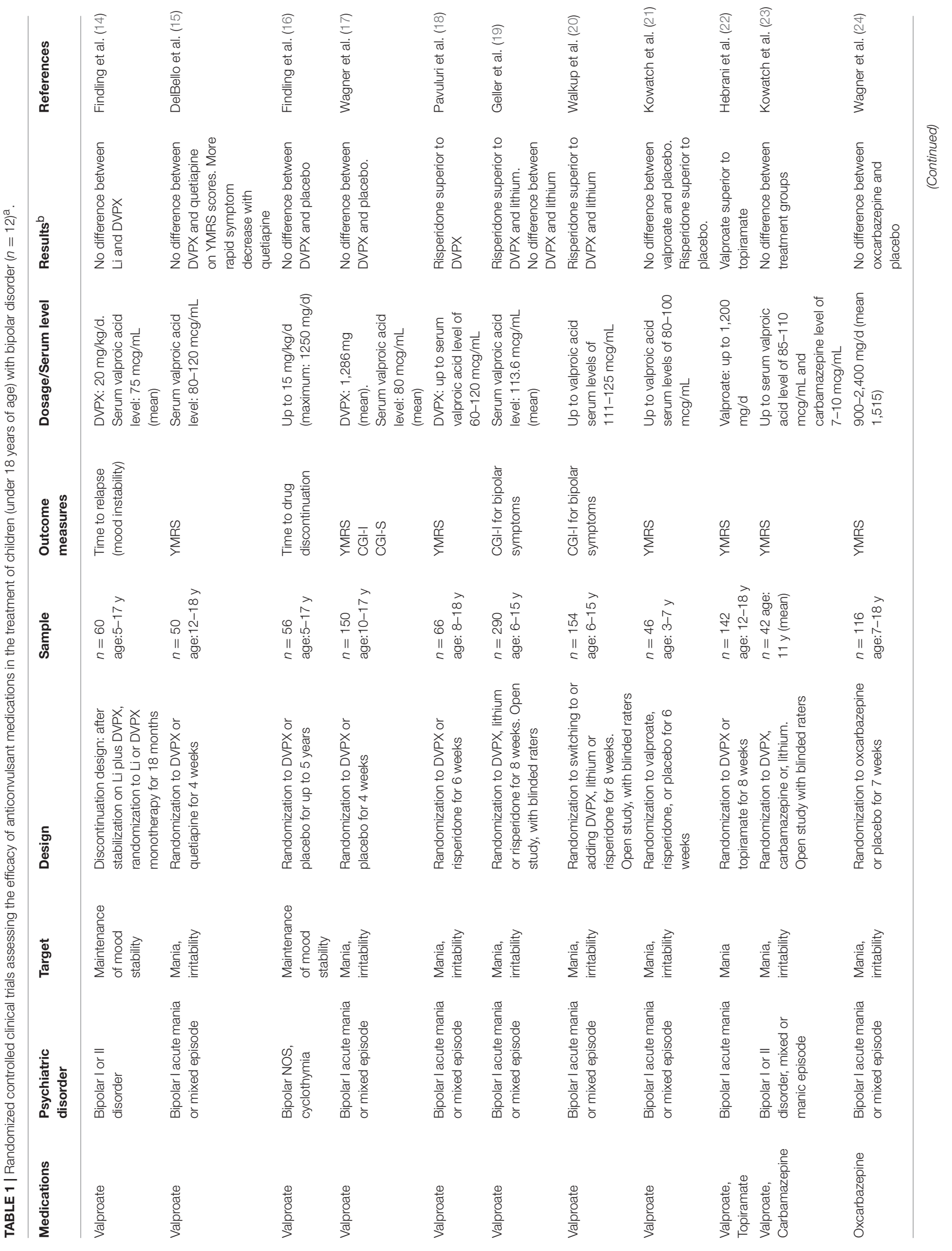




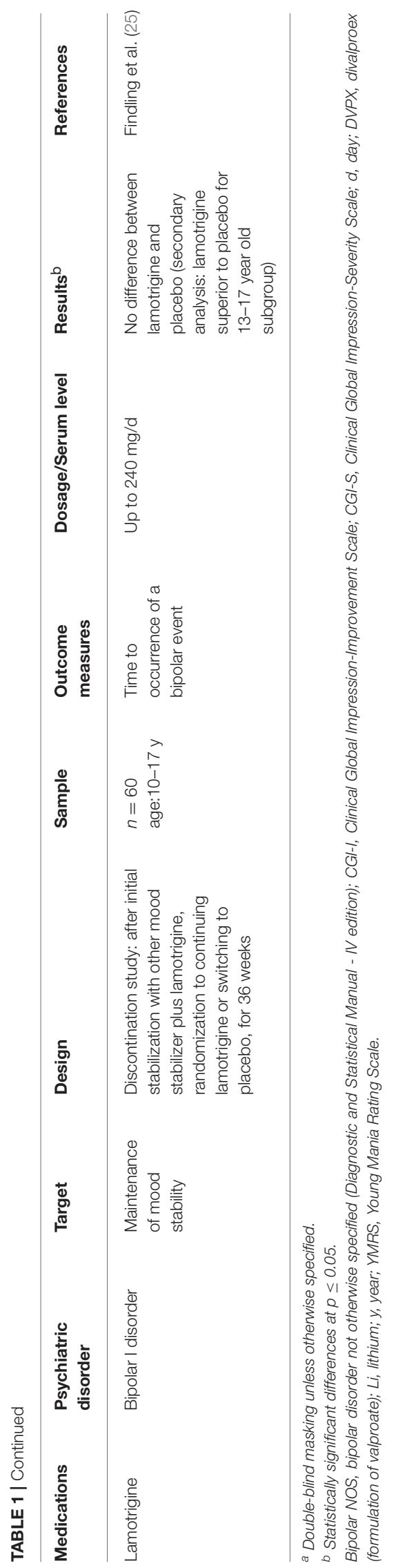

\section{DISCUSSION}

To evaluate the evidence for efficacy of anticonvulsant medications in psychiatric disorders of childhood, we conducted a systematic qualitative review of relevant published RCTs. We restricted the search to RCTs because uncontrolled studies cannot provide evidence of treatment effects given the variable placebo-response in psychiatric conditions.

Twenty-four RCTs met the pre-specified selection criteria (Tables 1, 2). The medication dosage and the duration of treatment were generally appropriate, and many studies measured medication serum levels. Most of these RCTs, however, had important methodological limitations, especially a small sample size ( $<40$ per treatment group), and therefore inadequate statistical power to detect medium effect sizes (see Supplementary Table 1).

A sample size of 40 subjects per treatment group will provide $80 \%$ statistical power to detect a between-group effect size usually considered in the medium range (e.g., a Cohen's $d=0.6)$ as statistically significant at a $p \leq 0.05$ (38). The small sample size of the large majority of these RCTs strongly limits their capacity to identify statistically significant treatment differences. In fact, out of 24 RCTs, 13 (54\%) did not find a statistically significant difference between the treatment groups.

A considerable number of RCTs was conducted on valproate $(n=15)$, three of which had adequate sample size, while few studies were devoted to other anticonvulsants. In the treatment of children with bipolar disorder, valproate showed no evidence of superiority over placebo, and was actually inferior to risperidone based on four RCT (Table 1).

Two RCTs compared valproate to lithium or quetiapine, respectively, in bipolar disorder, and found no difference between treatment groups $(14,15)$. Considering the small sample size of these RCTs and the lack of a placebo condition, the lack of difference cannot be interpreted as evidence of efficacy. Another, single-site, RCT compared topiramate to valproate in hospitalized, acutely manic youths (22). This study, which had been designed to test the efficacy of topiramate, found valproate to be superior to topiramate. The report, however, lacks an adequate description of the masking methods, and the especially large effect size is surprising and possibly due to the specific context of the hospital where the study was conducted.

These data on anticonvulsants in child bipolar disorder appear to be at odds with the evidence for efficacy of valproate, carbamazepine, and lamotrigine in adults with bipolar disorder. In adults, valproate and carbamazepine are superior to placebo in acute mania $(2,3)$, although less effective than antipsychotics (3), and lamotrigine is superior to placebo in bipolar depression (4). More limited evidence supports also the efficacy of valproate as maintenance treatment in adult bipolar disorder (5). The discrepancy between adults and children is suggestive of developmental differences in the psychopathology of the mood dysregulation. It should also be pointed out that there are several psychiatric medications, such as antidepressants and benzodiazepines, whose efficacy has been shown in adults but not in children (39). 


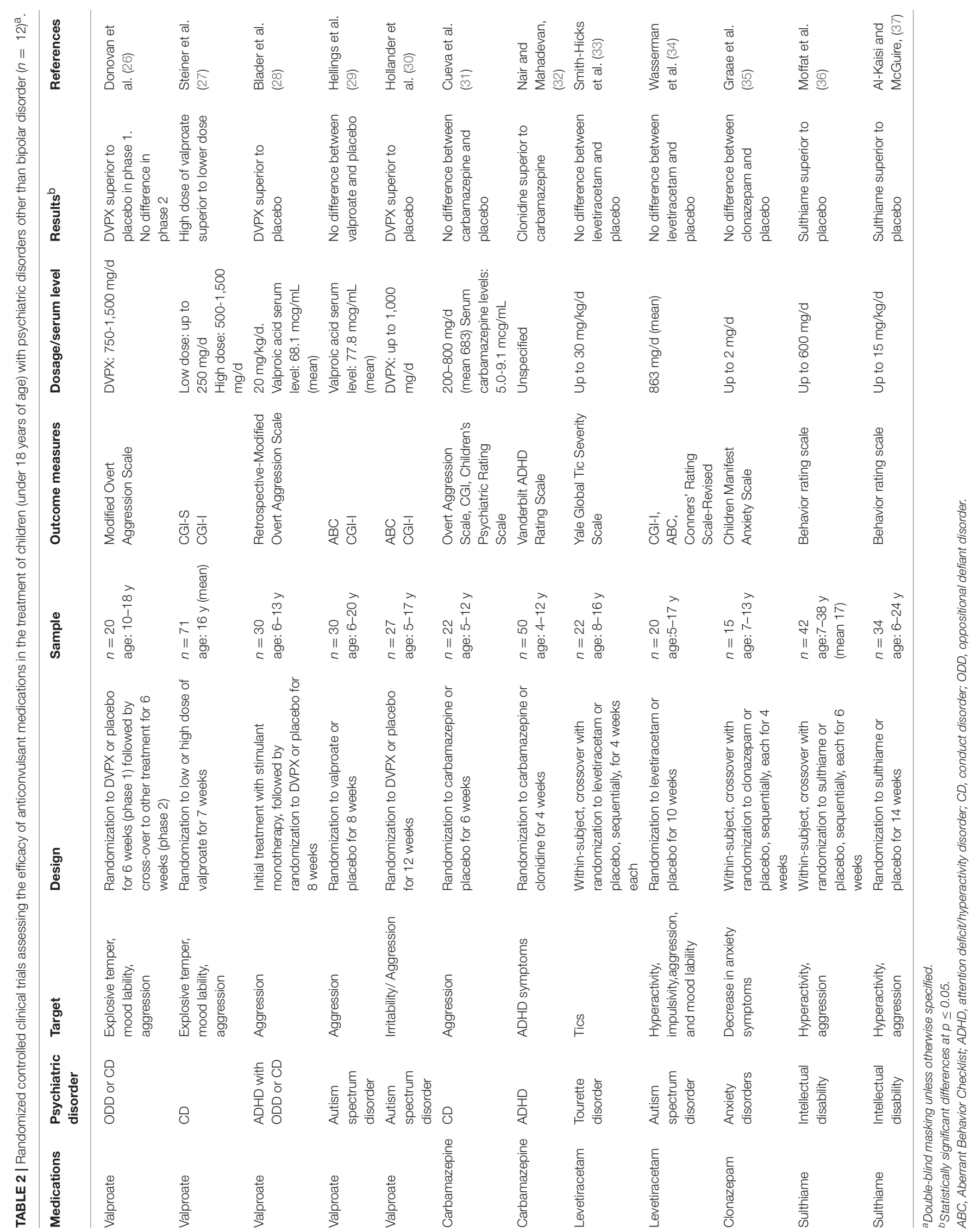


TABLE 3 | Primary target of the 24 randomized controlled clinical trials (RCTs) of anticonvulsant medications in psychiatric disorders in children (under 18 years of age).

\begin{tabular}{lc}
\hline & no of RCTs \\
\hline Control of acute symptoms of mania and irritability in bipolar disorder & 9 \\
Prevention of recurrent explosive aggression & 9 \\
Prevention of recurrence of bipolar acute episode & 3 \\
Control of symptoms of ADHD & 1 \\
Control of tics in tourette disorder & 1 \\
Control of symptoms of anxiety & 1 \\
\hline
\end{tabular}

For the management of aggression and explosive temper, valproate showed efficacy in four small and single-site RCTs in children with conduct disorder, ADHD, or autism (26-28, 30). One of these, used valproate as add-on treatment to stimulant medication in ADHD (28). Another one was designed as a crossover trial, but could not complete the second segment of the study and analyzed only the first part (26). Even if none of these studies met all the methodological quality criteria, these data can be taken as tentative evidence of efficacy of valproate in controlling aggressive behavior. This is consistent with the results of a meta-analysis of anticonvulsants in the management of aggression in adults (9). These findings, however, should be confirmed by adequately powered, multisite RCTs.

Little evidence of efficacy in bipolar disorder emerged from the RCTs of other commonly used anticonvulsants. In particular, a statistically powered, multi-site, placebo-controlled trial of oxcarbazepine in bipolar disorder found no statistically significant difference (24). Lamotrigine, which is effective in bipolar depression in adults, did no better than placebo in a RCT that included children aged from 10 to 17 years, although secondary analyses found superiority in the 13- to 17-year-old subgroup (26). Sulthiame, an infrequently used anticonvulsant, was found better than placebo in 2 RCTs conducted more than 40 years ago for the control of aggression and hyperactivity in

\section{REFERENCES}

1. Gobbi G, Janiri L. Sodium- and magnesium-valproate in vivo modulate glutamatergic and GABAergic synapses in the medial prefrontal cortex. Psychopharmacology (2006) 185:255-62. doi: 10.1007/s00213-006-0317-3

2. Macritchie K, Geddes JR, Scott J, Haslam D, de Lima M, Goodwin G. Valproate for acute mood episodes in bipolar disorder. Cochrane Database Syst Rev. (2003) CD004052. doi: 10.1002/14651858.CD004052

3. Cipriani A, Barbui C, Salanti G, Rendell J, Brown R, Stockton S, et al. Comparative efficacy and acceptability of antimanic drugs in acute mania: a multiple-treatments meta-analysis. Lancet (2011) 378:1306-15. doi: 10.1016/S0140-6736(11)60873-8

4. Geddes JR, Calabrese JR, Goodwin GM. Lamotrigine for treatment of bipolar depression: independent meta-analysis and meta-regression of individual patient data from five randomised trials. Br J Psychiatry (2009) 194:4-9. doi: 10.1192/bjp.bp.107.048504

5. Cipriani A, Reid K, Young AH, Macritchie K, Geddes J. Valproic acid, valproate and divalproex in the maintenance treatment of institutionalized patients, including both youths and adults, with severe intellectual disability $(36,37)$, but the implications of these data for current practice are unclear.

The limitations of this review are primarily related to the design characteristics of the studies and especially to the small sample size of most of them. The studies are also rather heterogeneous with respect to both the type of anticonvulsant being tested and the clinical indication being targeted. Another limitation is the relatively wide range of patient age in most studies, some of which included also adults.

Based on the current data, the psychiatric use of anticonvulsants in children cannot be supported according to evidence-based standards. Their potential benefit valproate and sulthiame for the management of recurrent aggression, however, cannot be discounted. The potential benefit of these medications must be in any case balanced against the risk for adverse effects, including psychiatric ones (40).

In conclusion, the efficacy of anticonvulsants as mood stabilizers in children with bipolar disorder remains unproven. There is limited evidence that some anticonvulsants may decrease aggressive behavior and explosive temper, especially in patients with neurodevelopmental disorders and intellectual disability. Because this evidence comes mainly from small studies, it might be informative to conduct more precisely designed and adequately powered RCT targeting recurrent and impulsive aggression in children with neurodevelopmental disorders.

\section{AUTHOR CONTRIBUTIONS}

$\mathrm{BV}$ and $\mathrm{CD}$ were responsible for review, data extraction, and evaluation. CC, RV, and MG contributed expert review and helped in the data interpretation and manuscript preparation.

\section{SUPPLEMENTARY MATERIAL}

The Supplementary Material for this article can be found online at: https://www.frontiersin.org/articles/10.3389/fpsyt. 2018.00270/full\#supplementary-material

bipolar disorder. Cochrane Database Syst Rev. (2013) CD003196. doi: 10.1002/14651858.CD003196.pub2

6. Pigott K, Galizia I, Vasudev K, Watson S, Geddes J, Young AH. Topiramate for acute affective episodes in bipolar disorder in adults. Cochrane Database Syst Rev. (2016) CD003384. doi: 10.1002/14651858.CD003384.pub3

7. Vasudev A, Macritchie K, Watson S, Geddes JR, Young AH. Oxcarbazepine in the maintenance treatment of bipolar disorder. Cochrane Database Syst Rev. (2008) CD005171. doi: 10.1002/14651858.CD005171.pub2

8. Vasudev A, Macritchie K, Vasudev K, Watson S, Geddes J, Young AH. Oxcarbazepine for acute affective episodes in bipolar disorder. Cochrane Database Syst Rev. (2001) CD004857. doi: 10.1002/14651858.CD004857.pub2

9. Jones RM, Arlidge J, Gillham R, Reagu S, van den Bree M, Taylor PJ. Efficacy of mood stabilisers in the treatment of impulsive or repetitive aggression: systematic review and meta-analysis. Br J Psychiatry (2011) 198:93-8. doi: 10.1192/bjp.bp.110.083030

10. Lopez-Larson M, Frazier JA. Empirical evidence for the use of lithium and anticonvulsants in children with psychiatric disorders. Harv Rev Psychiatry (2006) 14:285-304. doi: 10.1080/10673220601082869 
11. Bhangoo RK, Lowe CH, Myers FS, Treland J, Curran J, Towbin KE, et al. Medication use in children and adolescents treated in the community for bipolar disorder. J Child Adolesc Psychopharmacol. (2003) 13:515-22. doi: 10.1089/104454603322724904

12. Wagner KD, Weller EB, Carlson GA, Sachs G, Biederman J, Frazier JA, et al. An open-label trial of divalproex in children and adolescents with bipolar disorder. J Am Acad Child Adolesc Psychiatry (2002) 41:1224-30. doi: 10.1097/00004583-200210000-00012

13. Liberati A, Altman DG, Tetzlaff J, Mulrow C, Gøtzsche PC, Ioannidis JP, et al. The PRISMA statement for reporting systematic reviews and meta-analyses of studies that evaluate health care interventions: explanation and elaboration. PLoS Med. (2009) 6:e1000100. doi: 10.1371/journal.pmed.1000100

14. Findling RL, McNamara NK, Youngstrom EA, Stansbrey R, Gracious BL, Reed MD, et al. Double-blind 18-month trial of lithium versus divalproex maintenance treatment in pediatric bipolar disorder. J Am Acad Child Adolesc Psychiatry (2005) 44:409-17. doi: 10.1097/01.chi.0000155981.83865.ea

15. DelBello MP, Kowatch RA, Adler CM, Stanford KE, Welge JA, Barzman $\mathrm{DH}$, et al. A double-blind randomized pilot study comparing quetiapine and divalproex for adolescent mania. J Am Acad Child Adolesc Psychiatry (2006) 45:305-13. doi: 10.1097/01.chi.0000194567.63289.97

16. Findling RL, Frazier TW, Youngstrom EA, McNamara NK, Stansbrey RJ, Gracious BL, et al. Double-blind, placebo-controlled trial of divalproex monotherapy in the treatment of symptomatic youth at high risk for developing bipolar disorder. J Clin Psychiatry (2007) 68:781-8. doi: 10.4088/JCP.v68n0519

17. Wagner KD, Redden L, Kowatch RA, Wilens TE, Segal S, Chang K, et al. A double-blind, randomized, placebo-controlled trial of divalproex extended-release in the treatment of bipolar disorder in children and adolescents. J Am Acad Child Adolesc Psychiatry (2009) 48:519-32. doi: 10.1097/CHI.0b013e31819c55ec

18. Pavuluri MN, Henry DB, Findling RL, Parnes S, Carbray JA, Mohammed $\mathrm{T}$, et al. Double-blind randomized trial of risperidone versus divalproex in pediatric bipolar disorder. Bipolar Disord. (2010) 12:593-605. doi: 10.1111/j.1399-5618.2010.00850.x

19. Geller B, Luby JL, Joshi P, Wagner KD, Emslie G, Walkup JT, et al. A randomized controlled trial of risperidone, lithium, or divalproex sodium for initial treatment of bipolar I disorder, manic or mixed phase, in children and adolescents. Arch Gen Psychiatry (2012) 69:515-28. doi: 10.1001/archgenpsychiatry.2011.1508

20. Walkup JT, Wagner KD, Miller L, Yenokyan G, Luby JL, Joshi PT, et al. Treatment of early-age mania: outcomes for partial and nonresponders to initial treatment. J Am Acad Child Adolesc Psychiatry (2015) 54:1008-19. doi: 10.1016/j.jaac.2015.09.015

21. Kowatch RA, Scheffer RE, Monroe E, Delgado S, Altaye M, Lagory D. Placebocontrolled trial of valproic acid versus risperidone in children 3-7 years of age with bipolar I disorder. J Child Adolesc Psychopharmacol. (2015) 25:306-13. doi: 10.1089/cap.2014.0166

22. Hebrani P, Behdani F, Manteghi AA. Double-blind, randomized, clinical trial of topiramate versus sodium valproate for the treatment of bipolar disorder in adolescents. Pak J Med Sci. (2009) 25:247-52.

23. Kowatch RA, Suppes T, Carmody TJ, Bucci JP, Hume JH, Kromelis M, et al. Effect size of lithium, divalproex sodium, and carbamazepine in children and adolescents with bipolar disorder. J Am Acad Child Adolesc Psychiatry (2000) 39:713-20. doi: 10.1097/00004583-200006000-00009

24. Wagner KD, Kowatch RA, Emslie GJ, Findling RL, Wilens TE, McCague K, et al. A double-blind, randomized, placebo-controlled trial of oxcarbazepine in the treatment of bipolar disorder in children and adolescents. Am J Psychiatry (2006)163:1179-86. doi: 10.1176/ajp.2006.163.7.1179

25. Findling RL, Chang K, Robb A, Foster VJ, Horrigan J, Krishen A, et al. Adjunctive maintenance lamotrigine for pediatric bipolar I disorder: a placebo-controlled, randomized withdrawal study. J Am Acad Child Adolesc Psychiatry (2015) 54:1020-31. doi: 10.1016/j.jaac.2015.09.017
26. Donovan SJ, Stewart JW, Nunes EV, Quitkin FM, Parides M, Daniel W, et al. Divalproex treatment for youth with explosive temper and mood lability: a double-blind, placebo-controlled crossover design. Am J Psychiatry (2000) 157:818-20. doi: 10.1176/appi.ajp.157.5.818

27. Steiner H, Petersen ML, Saxena K, Ford S, Matthews Z. Divalproex sodium for the treatment of conduct disorder: a randomized controlled clinical trial. $J$ Clin Psychiatry (2003) 64:1183-91. doi: 10.4088/JCP.v64n1007

28. Blader JC, Schooler NR, Jensen PS, Pliszka SR, Kafantaris V. Adjunctive divalproex versus placebo for children with ADHD and aggression refractory to stimulant monotherapy. Am J Psychiatry (2009) 166:1392-401. doi: 10.1176/appi.ajp.2009.09020233

29. Hellings JA, Weckbaugh M, Nickel EJ, Cain SE, Zarcone JR, Reese RM, et al. A double-blind, placebo-controlled study of valproate for aggression in youth with pervasive developmental disorders. J Child Adolesc Psychopharmacol. (2005) 15:682-92. doi: 10.1089/cap.2005.15.682

30. Hollander E, Chaplin W, Soorya L, Wasserman S, Novotny S, Rusoff J, et al. Divalproex sodium vs placebo for the treatment of irritability in children and adolescents with autism spectrum disorders. Neuropsychopharmacology (2010) 35:990-8. doi: 10.1038/npp.2009.202

31. Cueva JE, Overall JE, Small AM, Armenteros JL, Perry R, Campbell M. Carbamazepine in aggressive children with conduct disorder: a double-blind and placebo-controlled study. J Am Acad Child Adolesc Psychiatry (1996) 35:480-90. doi: 10.1097/00004583-199604000-00014

32. Nair V, Mahadevan S. Randomised controlled study-efficacy of clonidine versus carbamazepine in children with ADHD. J Trop Pediatr. (2009) 55:11621. doi: 10.1093/tropej/fmn117

33. Smith-Hicks CL, Bridges DD, Paynter NP, Singer HS. A double blind randomized placebo control trial of levetiracetam in Tourette syndrome. Mov Disord. (2007) 22:1764-70. doi: 10.1002/mds.21615

34. Wasserman S, Iyengar R, Chaplin WF, Watner D, Waldoks SE, Anagnostou $\mathrm{E}$, et al. Levetiracetam versus placebo in childhood and adolescent autism: a double-blind placebo-controlled study. Int Clin Psychopharmacol. (2006) 21:363-7. doi: 10.1097/01.yic.0000224787.13782.0f

35. Graae F, Milner J, Rizzotto L, Klein RG. Clonazepam in childhood anxiety disorders. J Am Acad Child Adolesc Psychiatry (1994) 33:372-6. doi: 10.1097/00004583-199403000-00011

36. Moffatt WR, Siddiqui AR, MacKay DN. The use of sulthiame with disturbed mentally subnormal patients. Br J Psychiatry (1970) 117:673-8. doi: 10.1192/bjp.117.541.673

37. Al Kaisi AH, McGuire RJ. The effect of sulthiame on disturbed behaviour in mentally subnormal patients. Br J Psychiatry (1974) 124:45-9. doi: 10.1192/bjp.124.1.45

38. Cohen J. Statistical Power Analysis for the Behavioral Sciences. Hillsdale, NJ: Lawrence Erlbaum Associates (1988).

39. Vitiello B, Ordó-ez AE. Pharmacological treatment of children and adolescents with depression. Expert Opin Pharmacother. (2016) 17:2273-9. doi: 10.1080/14656566.2016.1244530

40. Brodie MJ, Besag F, Ettinger AB, Mula M, Gobbi G, Comai S, et al. Epilepsy, antiepileptic drugs, and aggression: an evidence-based review. Pharmacol Rev. (2016) 68:563-602. doi: 10.1124/pr.115.012021

Conflict of Interest Statement: The authors declare that the research was conducted in the absence of any commercial or financial relationships that could be construed as a potential conflict of interest.

Copyright (c) 2018 Davico, Canavese, Vittorini, Gandione and Vitiello. This is an open-access article distributed under the terms of the Creative Commons Attribution License (CC BY). The use, distribution or reproduction in other forums is permitted provided the original author(s) and the copyright owner are credited and that the original publication in this journal is cited, in accordance with accepted academic practice. No use, distribution or reproduction is permitted which does not comply with these terms. 